\title{
Effects of Isoflurane Concentration on Basic Echocardiographic Parameters of the Left Ventricle in Rats
}

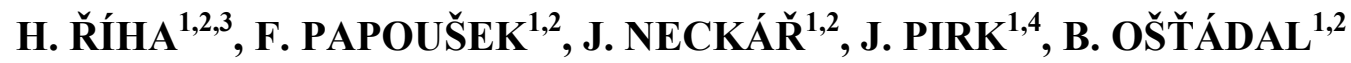 \\ ${ }^{1}$ Centre for Cardiovascular Research, Prague, Czech Republic, ${ }^{2}$ Institute of Physiology, Academy \\ of Sciences of the Czech Republic, Prague, Czech Republic, ${ }^{3}$ Department of Anesthesiology and \\ Intensive Care Medicine, Institute for Clinical and Experimental Medicine, Prague, Czech \\ Republic, ${ }^{4}$ Department of Cardiovascular Surgery, Institute for Clinical and Experimental \\ Medicine, Prague, Czech Republic
}

Received October 2, 2011

Accepted May 11, 2012

On-line June 6, 2012

\section{Summary}

Transthoracic echocardiography (TTE) has become an important modality for the assessment of cardiac structure and function in animal experiments. The acquisition of echocardiographic images in rats requires sedation/anesthesia to keep the rats immobile. Commonly used anesthetic regimens include intraperitoneal or inhalational application of various anesthetics. Several studies have compared the effects of anesthetic agents on echocardiographic parameters in rats; however, none of them examined the effects of different concentrations of inhalational anesthetics on echocardiographic parameters. Accordingly, the aim of this study was to examine the effects of different concentrations of isoflurane used for anesthesia during TTE examination in rats on basic echocardiographic parameters of left ventricular (LV) anatomy and systolic function. TTE examinations were performed in adult male Wistar rats $(n=10)$ anesthetized with isoflurane at concentrations of $1.5-3 \%$. Standard echocardiograms were recorded for off-line analysis. An absence of changes in basic echocardiographic parameters of LV anatomy and systolic function was found under isoflurane anesthesia using concentrations between 1.5-2.5 \%. An isoflurane concentration of $3 \%$ caused a small, but statistically significant, increase in LV chamber dimensions without a concomitant change in heart rate or fractional shortening. For the purpose of TTE examination in the rat, our results suggest that isoflurane concentrations $\leq 2.5 \%$ can be safely recommended.

\section{Key words}

Anesthesia - Isoflurane • Echocardiography • Left ventricular function $\bullet$ Rat heart

\section{Corresponding author}

H. Ř́ha, Department of Anesthesiology and Intensive Care Medicine, Institute for Clinical and Experimental Medicine, Vídeňská 1958/9, 14021 Prague, Czech Republic. Fax: +42026136 2799. E-mail: hynek.riha@ikem.cz

Transthoracic echocardiography (TTE) has become an important modality for the assessment of cardiac structure and function in many animal models of cardiac diseases. TTE is a noninvasive and quantitative method which can be easily repeated. Rats are commonly used as models of cardiac diseases because they are inexpensive, and microsurgical procedures on their hearts (e.g., coronary artery ligation) have been wellestablished.

The acquisition of echocardiographic images of an adequate quality within a short period of time requires sedation/anesthesia to immobilize the rat during examination. Typical anesthetic regimens include intraperitoneal injection of various anesthetics (e.g., pentobarbital, tribromoethanol and ketamine with xylazine or midazolam) or the application of inhalational anesthetics (Plante et al. 2006, Stein et al. 2007). Anesthetic agents are well-known to exert various effects 
on many parameters of the cardiovascular system (e.g., heart rate, ventricular contractility and blood pressure), and in most cases, there is a discernable dose-response relationship (Mushambi and Smith 2007, Smith 2009).

In previous animal studies, significant differences in echocardiographic parameters measured under the influence of different anesthetics were found. The effects of anesthetic agents on echocardiographic parameters have been reported in detail in mice (Chaves et al. 2001, Hart et al. 2001, Roth et al. 2002); the influence of increasing concentrations of inhalational anesthetics was also studied (Gentry-Smetana et al. 2008). A few studies have compared the effects of different anesthetic agents used for TTE on echocardiographic parameters in rats (Plante et al. 2006, Stein et al. 2007); however, none of these studies examined the effects of different concentrations of inhalational anesthetics. Among published studies, the most frequently reported inhalational anesthetic used for TTE in rats was isoflurane at concentrations ranging from $1.5 \%$ (Plante et al. 2006, Stein et al. 2007) to $2 \%$ (Liu and Rigel 2009).

The rat is considered a better model for human cardiovascular diseases than the mouse (Abbott 2004). Despite their similarity, there are distinct anatomical, physiological, cellular and genetic differences between rats and mice. For example, the murine heart has a significantly higher heart rate $(500-700 / \mathrm{min})$ than the rat heart (300-400/min) (Gross 1994). The cell composition of their cardiac muscles is also significantly different; $\sim 56 \%$ of the cells are myocytes and $\sim 27 \%$ of the cells are fibroblasts in the murine heart, whereas only $\sim 30 \%$ of the cells are myocytes in the rat heart, which is accompanied by a higher number $(\sim 64 \%)$ of fibroblasts (Banerjee et al. 2007).

Accordingly, the main objective of our study was to investigate the effects of different concentrations of isoflurane administered during TTE examination in rats on basic echocardiographic parameters of left ventricular (LV) anatomy and systolic function.

This experimental protocol was approved by the Animal Care and Use Committee of the Institute of Physiology, Academy of Sciences of the Czech Republic. Experiments were conducted in accordance with the Guide for the Care and Use of Laboratory Animals published by the US National Institutes of Health (NIH publication No. 85-23, revised 1996). Adult male Wistar rats (body weight [BW] 262-290 g) were used ( $\mathrm{n}=10)$.
Anesthesia was induced by placing each rat in an induction chamber (calibrated closed-box system with a variable bypass vaporizer) filled with $2.5 \%$ isoflurane at a flow rate of $3 \mathrm{l} / \mathrm{min}$ for $3 \mathrm{~min}$. During the next $5 \mathrm{~min}$, the rat was placed on a warming pad $\left(38^{\circ} \mathrm{C}\right)$ and shaved from the left sternal border to the left axillary line. The gold-plated electrodes of the warming pad were attached to the rat's legs, and pre-warmed ultrasound gel was applied to the chest wall. The TTE examination began $8 \mathrm{~min}$ after the induction of anesthesia. Anesthesia during the preparatory phase and TTE examination was maintained by applying a given inspiratory concentration (1.5-3\%) of isoflurane at a flow rate of $2 \mathrm{l} / \mathrm{min}$ through a sealed nose cone during spontaneous breathing. TTE examinations were performed under the different isoflurane concentrations studied on different days to avoid potential residual effects of isoflurane.

All studies were performed by an experienced echocardiographer using a 14-MHz matrix probe (M12L) coupled to a GE Medical Vivid 7 Dimension System (GE Medical Systems CZ Ltd., Prague, CZ). Standard echocardiograms (Liu and Rigel 2009) were recorded and stored digitally for off-line analysis. Images were analyzed with Echo PAC PC version 7.1.2 software (GE Medical Systems CZ Ltd., Prague, CZ).

Directly measured parameters included LV enddiastolic and end-systolic anterior and posterior wall thickness, and LV chamber end-diastolic (EDD) and endsystolic diameter (ESD). From the measurements of LV chamber dimensions, fractional shortening (FS) and enddiastolic volume (EDV) by the Teichholz formula (Teichholz et al. 1976) were derived: FS (\%) = [(EDD$\mathrm{ESD}) / \mathrm{EDD}] \times 100$, and $\mathrm{EDV}=[7.0 /(2.4+\mathrm{EDD})] \times \mathrm{EDD}^{3}$. Relative LV wall thickness (RWT) was defined as the sum of end-diastolic anterior wall + posterior wall thickness, divided by EDD. Values of heart rate (HR) were averaged from all evaluated loops under each particular isoflurane concentration.

Data are expressed as means \pm S.E.M. Repeated measures analysis of variance (ANOVA) was used to analyze differences among particular isoflurane concentrations. If the findings from the ANOVA were significant, the Newman-Keuls post-hoc test was employed. Differences were considered significant when $P<0.05$. All statistical analyses were performed using GraphPad Prism version 5.04 software for Windows (GraphPad Software, San Diego, CA, USA). 
Table 1. Echocardiographic left ventricular anatomical parameters under different isoflurane concentrations.

\begin{tabular}{lcccr}
\hline & $\mathbf{1 . 5} \%$ ISO & $\mathbf{2 . 0} \%$ ISO & $\mathbf{2 . 5} \%$ ISO & 3.0 \% ISO \\
\hline AWTd $(\mathrm{mm})$ & $1.5 \pm 0.02$ & $1.5 \pm 0.03$ & $1.5 \pm 0.02$ & $1.5 \pm 0.03$ \\
AWTs $(\mathrm{mm})$ & $2.5 \pm 0.05$ & $2.5 \pm 0.06$ & $2.4 \pm 0.05$ & $2.4 \pm 0.06$ \\
PWTd $(\mathrm{mm})$ & $1.7 \pm 0.05$ & $1.7 \pm 0.05$ & $1.6 \pm 0.05$ & $1.6 \pm 0.04$ \\
$P W T s(\mathrm{~mm})$ & $2.7 \pm 0.04$ & $2.7 \pm 0.05$ & $2.6 \pm 0.05$ & $2.6 \pm 0.05$ \\
$R W T$ & $0.47 \pm 0.015$ & $0.47 \pm 0.019$ & $0.45 \pm 0.012$ & $0.43 \pm 0.011$ \\
$E D D(\mathrm{~mm})$ & $6.7 \pm 0.12$ & $6.7 \pm 0.14$ & $6.8 \pm 0.11$ & $7.0 \pm 0.12^{*}$ \\
$E S D(\mathrm{~mm})$ & $3.8 \pm 0.11$ & $3.8 \pm 0.13$ & $3.9 \pm 0.11$ & $4.0 \pm 0.13^{*}$ \\
$E D V(\mu \mathrm{l})$ & $233.8 \pm 8.64$ & $239.5 \pm 10.68$ & $242.7 \pm 8.28$ & $258.4 \pm 7.79^{*}$ \\
\hline
\end{tabular}

Values are presented as means \pm S.E.M. ${ }^{*} P<0.05$ vs. lower isoflurane concentrations $(1.5 \%, 2.0 \%, 2.5 \%)$. ISO, isoflurane; AWTd, anterior wall thickness in diastole; AWTs, anterior wall thickness in systole; PWTd, posterior wall thickness in diastole; PWTs, posterior wall thickness in systole; RWT, relative wall thickness; EDD, end-diastolic diameter; ESD, end-systolic diameter; EDV, end-diastolic volume.
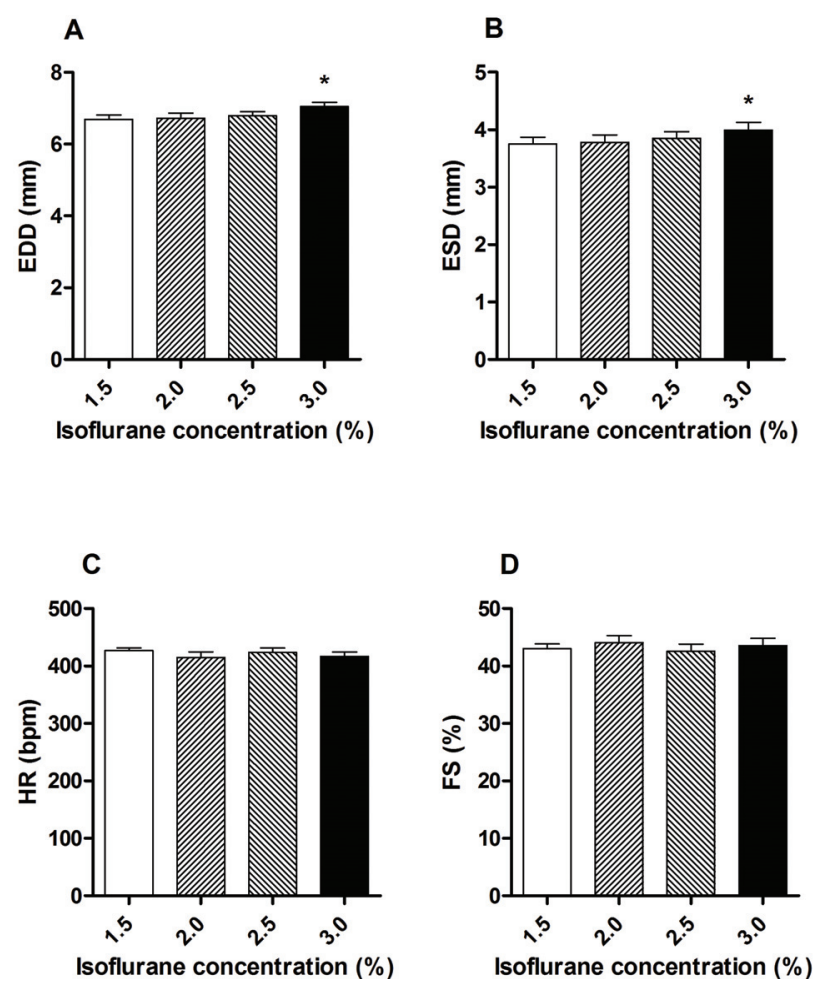

Fig. 1. Echocardiographic parameters of left ventricular chamber dimensions and systolic function under different isoflurane concentrations. Values are means \pm S.E.M. ${ }^{*} P<0.05$ vs. lower isoflurane concentrations (1.5\%, $2.0 \%, 2.5 \%)$. A: End-diastolic diameter (EDD). B: End-systolic diameter (ESD). C: Heart rate $(\mathrm{HR})$; beats per minute (bpm). D: Fractional shortening (FS).

The thickness of the LV anterior and posterior walls during systole and diastole as well as RWT was not influenced by isoflurane concentration (Table 1). Increasing the isoflurane concentration from $1.5 \%$ to $2.5 \%$ did not change the EDD (Fig. 1A); however, under anesthesia with $3 \%$ isoflurane, the EDD was higher when compared to lower isoflurane concentrations
$(P=0.0007)$. An identical relationship was observed for the ESD (Fig. 1B) with values recorded under a $3 \%$ isoflurane concentration being higher than under lower isoflurane concentrations $(P=0.0008)$. The EDV derived from the EDD measurements (Table 1) also followed the same trend with higher values under a $3 \%$ isoflurane concentration $(P=0.0022)$.

Average HR values were comparable among the groups (Fig. 1C). Similarly, the FS did not change under different isoflurane concentrations (Fig. 1D).

The primary finding of this study is an absence of changes in the basic echocardiographic parameters of LV anatomy and systolic function under isoflurane anesthesia at concentrations between 1.5-2.5\%. The highest isoflurane concentration ( $3 \%$ ) caused a small, but statistically significant, increase in LV end-diastolic and end-systolic diameters and EDV without a concomitant change in HR or FS.

Experimental studies performed in rats have demonstrated significant differences among the effects of different anesthetics on echocardiographic parameters. In healthy rats, HR and cardiac output remained higher and closer to normal values under isoflurane anesthesia as compared to ketamine/xylazine, while LV chamber dimensions and systolic function were not significantly affected by the choice of anesthetic (Plante et al. 2006). Another study examining echocardiographic parameters in conscious rats and under anesthesia with pentobarbital, isoflurane or ketamine/xylazine documented a decrease in diastolic anterior wall thickness and an increase in EDD and EDV by isoflurane as compared to the conscious state. In that study, isoflurane also caused a decrease in LV systolic function compared to the conscious state 
(Stein et al. 2007).

The only study investigating the effects of different concentrations $(0.5,1,2,3,4$ and $5 \%)$ of the inhalational anesthetics isoflurane and sevoflurane on echocardiographic parameters was performed in mice and concerned primarily LV functional parameters (GentrySmetana et al. 2008). The results included a decrease in FS with concentrations of isoflurane $\geq 4 \%$, a decrease in ejection fraction with concentrations $\geq 3 \%$, and an increase in EDV at a $4 \%$ concentration of isoflurane. The HR values did not vary with the changes in isoflurane concentration. Sevoflurane, compared to isoflurane, caused more profound depressions of LV systolic function and HR even with concentrations $\geq 2 \%$.

The results of our study in rats are similar to those of prior study in mice. All of studied isoflurane concentrations (i.e., 1.5-3\%) in rats maintained HR and LV systolic function, expressed as FS, unchanged. Only a $3 \%$ isoflurane concentration induced changes in ESD, EDD and EDV, whereas under lower concentrations LV chamber dimensions did not differ. Important differences in the comparison with prior study in mice include the difference in the sex of employed animals (male rats vs. female mice), and a significant increase in EDV at a $3 \%$ isoflurane concentration in rats vs. a $4 \%$ concentration in mice.

A possible limitation of our study is that we did not examine isoflurane concentrations greater than $3 \%$; we cannot exclude that concentrations above studied range would bring different results. We chose to study commonly reported concentrations (i.e., 1.5-2 \%) used in rats for TTE examination with two concentrations above this range (2.5-3\%). Isoflurane concentrations $\leq 2 \mathrm{MAC}$ (minimal alveolar concentration), when $1 \mathrm{MAC}$ corresponds to $1.3 \%$ concentration of isoflurane (Rampil et al. 2001), are fully sufficient to immobilize rats during TTE examination. Also, we did not investigate other echocardiographic parameters of LV chamber dimensions and function (e.g., end-diastolic and end-systolic area with fractional area change); rather, this study was focused on the most important and frequently reported parameters. Furthemore, we applied strict time protocol during the study with TTE examination beginning exactly $8 \mathrm{~min}$ after the induction of anesthesia; we cannot exclude that prolonged isoflurane administration could bring different results.

It is important to acknowledge that the absolute differences in LV chamber dimensions between $3 \%$ and lower concentrations of isoflurane, despite their statistical significance, are of relatively small magnitude. Furthemore, the present study included only normal rats without impairments of cardiovascular system; therefore, the generalizability of these results to the various rat models of cardiac diseases is unknown.

In conclusion, we demonstrated that under isoflurane concentrations between 1.5-2.5 \%, used for anesthesia during TTE examination in rats, the basic echocardiographic parameters of LV chamber anatomy and systolic function were not different. At concentrations above this range, isoflurane appears to significantly influence echocardiographic parameters of LV chamber dimensions. Our study confirmed the results in mice; significant isoflurane-induced changes in basic echocardiographic parameters of the left ventricle in small rodents commence with higher isoflurane concentrations. For the purpose of TTE examination in rats, our results suggest that isoflurane concentrations $\leq 2.5 \%$ can be safely recommended.

\section{Conflict of Interest}

There is no conflict of interest.

\section{Acknowledgements}

The study was supported by grants from the Ministry of Education of the Czech Republic (MSMT 1M0510) and the Grant Agency of the Academy of Sciences of the Czech Republic (IAA 501110901).

\section{References}

ABBOTT A: Laboratory animals: the Renaissance rat. Nature 428: 464-466, 2004.

BANERJEE I, FUSELER JW, PRICE RL, BORG TK, BAUDINO TA: Determination of cell types and numbers during cardiac development in the neonatal and adult rat and mouse. Am J Physiol Heart Circ Physiol 293: H1883H1891, 2007.

CHAVES AA, WEINSTEIN DM, BAUER JA: Non-invasive echocardiographic studies in mice: influence of anesthetic regimen. Life Sci 69: 213-222, 2001. 
GENTRY-SMETANA S, REDFORD D, MOORE D, LARSON DF: Direct effects of volatile anesthetics on cardiac function. Perfusion 23: 43-47, 2008.

GROSS DR: Animal models in cardiovascular research. 2nd revised ed., Kluwer Academic Publishers, Dordrecht, Boston 1994.

HART CY, BURNETT JC JR, REDFIELD MM: Effects of avertin versus xylazine-ketamine anesthesia on cardiac function in normal mice. Am J Physiol Heart Circ Physiol 281: H1938-H1945, 2001.

LIU J, RIGEL DF: Echocardiographic examination in rats and mice. Methods Mol Biol 573: 139-155, 2009.

MUSHAMBI MC, SMITH G: Inhalational anaesthetic agents. In: Textbook of Anaesthesia. AR AITKENHEAD, G SMITH, DJ ROWBOTHAM (eds), 5th ed., Churchill Livingstone Elsevier, Edinburgh, 2007, pp 13-33.

PLANTE E, LACHANCE D, ROUSSEL E, DROLET MC, ARSENAULT M, COUET J: Impact of anesthesia on echocardiographic evaluation of systolic and diastolic function in rats. $J$ Am Soc Echocardiogr 19: 1520-1525, 2006.

RAMPIL IJ, LASTER MJ, EGER EI 2ND: Antagonism of the 5-HT(3) receptor does not alter isoflurane MAC in rats. Anesthesiology 95: 562-564, 2001.

ROTH DM, SWANEY JS, DALTON ND, GILPIN EA, ROSS J JR: Impact of anesthesia on cardiac function during echocardiography in mice. Am J Physiol Heart Circ Physiol 282: H2134-H2140, 2002.

SMITH TC: Hypnotics and intravenous anaesthetic agents. In: Fundamentals of Anaesthesia. T SMITH, C PINNOCK, T LIN (eds), 3rd ed., Cambridge University Press, Cambridge, 2009, pp 569-583.

STEIN AB, TIWARI S, THOMAS P, HUNT G, LEVENT C, STODDARD MF, TANG XL, BOLLI R, DAWN B: Effects of anesthesia on echocardiographic assessment of left ventricular structure and function in rats. Basic Res Cardiol 102: 28-41, 2007.

TEICHHOLZ LE, KREULEN T, HERMAN MV, GORLIN R: Problems in echocardiographic volume determinations: echocardiographic-angiographic correlations in the presence of absence of asynergy. Am J Cardiol 37: 7-11, 1976. 